\title{
Effect of pine bark (Pinus radiata) extracts on sporulation of coccidian oocysts
}

\author{
Abdul Lateef Molan, Zhuojian Liu and Shampa De
}

Division of Human Health and Physiology, Institute of Food, Nutrition and Human Health, Massey University, Palmerston North, New Zealand

\begin{abstract}
A series of experiments have been undertaken to determine the effect of water extracts from pine bark (Pinus radiata) on the inhibition of the sporulation of oocysts of three species of avian coccidia. Tubes containing coccidian oocysts isolated from droppings of coccidia-infected chickens were randomly assigned to $0,250,500$ and $1000 \mu \mathrm{g} / \mathrm{ml}$ pine bark extract (PBE). The tubes were incubated at $25-29{ }^{\circ} \mathrm{C}$ for $48 \mathrm{~h}$ depending on the species of Eimeria. Sporulation inhibition bioassay was used to evaluate the activity of PBE on the sporulation of coccidian oocysts. The oocysts were gently aerated with an air pump away from sun light. The results show for the first time that water-soluble extracts from pine bark containing $35 \%$ condensed tannins have anticoccidial activity as evidenced by their ability to decrease significantly the sporulation of the oocysts of three species of Eimeria, namely Eimeria tenella (Railliet et Lucet, 1891), E. maxima Tyzzer, 1929 and E. acervulina Tyzzer, 1929, under laboratory conditions. Incubation of unsporulated oocysts of these parasites in water containing $500 \mu \mathrm{g}$ PBE per ml resulted in inhibition of sporulation of these oocysts by about $28-84 \%$ relative to the oocysts in the control incubations. In addition, up to $12 \%$ of E. maxima oocysts exposed to 500-1000 $\mu \mathrm{g}$ pine bark/ml were containing abnormal sporocysts in terms of size, number and shape.
\end{abstract}

Key words: Eimeria acervulina, Eimeria tenella, Eimeria maxima, pine bark, Pinus radiata, sporulation, anticoccidial activity

Coccidiosis is the major parasitic disease of poultry. It is considered to be one of the most economically important diseases of domestic poultry throughout the world (Allen and Fetterer 2002). Avian coccidiosis is a very harmful disease in the poultry industry of many countries. This disease costs the US poultry industry, for example, more than $\$ 1.5$ billion in annual losses (Yun et al. 2000).

The effective use of anticoccidial drugs over the past 50 years has, in part, provided the basis for the rapid growth of the poultry industry and the increased availability of high quality, affordable poultry products to the consumers. However, the increasing resistance of avian coccidia to currently used anticoccidial drugs (Williams 2006), coupled with the increasing costs of developing new drugs and the public's distrust of drug-treated meat demonstrate the urgent need to explore alternative methods of controlling this disease.

It seems that avian coccidial parasites (Eimeria spp.) are highly immunogenic as the primary infections can stimulate solid immunity to homologous challenges (Allen and Fetterer 2002). Some studies suggest that vaccines could offer excellent alternative strategies to drugs for controlling coccidiosis (Lillehoj et al. 2000). Several vaccines, especially those based on live and attenuated strains of coccidians have been marketed (Allen and Fetterer 2002, Waldenstedt 2003).
Pinus radiata D. Don (Coniferopsida: Pinaceae), also known as Monterey pine and commonly referred to as radiata pine, is native to the coast of southern California, USA and Mexico (Millar 1999). In some countries including New Zealand, Australia, Chile and South Africa, radiata pine is grown extensively as an exotic timber species (Scott 1960). Moreover, P. radiata is grown in northern hemisphere countries including Spain, Portugal, Greece, Turkey, Italy, and Russia (Scott 1960). Pine bark extract (PBE) is a unique mixture of flavonoids, which comprises $8 \%(\mathrm{w} / \mathrm{w})$ of flavonoid monomers and $85 \%(\mathrm{w} / \mathrm{w})$ of condensed tannins (procyanidins) (Rice-Evans et al. 1996). Phenolic contents of pine bark are catechin, epicatechin, quercetin, dihydroquercetin, taxifolin, phenolic acids, and procyanidin dimers, trimers, oligomers, and polymers formed from catechin and epicatechin (Porter 1974, Packer et al. 1999). Pine bark extract has been used in traditional medicine in Europe and North America for long time for many diseases and illnesses (Packer et al. 1999). Kim et al. (2004) reported that pine bark extracts have high inhibition activity against several carbohydrate-hydrolyzing enzymes. Recently, Kim et al. (2005) investigated the inhibitory effect of PBE on carbohydrate-hydrolyzing enzymes and the hypoglycaemic effect in diabetic mice. The authors found that PBE showed inhibitory effect against salivary $\alpha$-amylase and yeast $\alpha$-glucosidase. Furthermore, 
in animal experiments PBE effectively suppressed the increase of postprandial blood glucose level by delaying absorption of diet, and body weights. More recently, Ahn et al. (2007) reported that pine bark extract (Pycnogenol) effectively reduced the number of pathogens and retarded the formation of thiobarbituric acid-related substances in raw beef.

This study is a part of a programme aiming to find alternative strategies to treat coccidiosis in chicken using polyphenols-containing forages and medicinal plants. The potential of using pine bark in livestock management is, however, not documented. The objective of this study was to measure the effects of Pinus radiata bark extract on the sporulation of the oocysts of three species of Eimeria, E. tenella (Railliet et Lucet, 1891), E. maxima Tyzzer, 1929 and E. acervulina Tyzzer, 1929.

\section{MATERIALS AND METHODS}

Preparation of pine bark extract and measurement of condensed tannins (CT). Pinus radiata bark was obtained from 7-10 year old trees grown in Manawatu area, New Zealand. The bark was fed through a wood chipper two times, and then ground in a Wiley ${ }^{\mathrm{TM}}$ mill through a $2-\mathrm{mm}$ mesh. The ground bark was further reduced in size in a Cyclotech (Foss Tecator ${ }^{\mathrm{TM}}$ ) rotary grinder to yield a fine powder. The finely ground bark was mixed with boiling water in a 1:10 ratio, stirred at $3-5$ min intervals for approximately $20 \mathrm{~min}$, and poured through a double layer of cheesecloth to remove particulate matter. This process was carried out with $500 \mathrm{~g}$ of powder made up to 5 litres with water to yield about 3.5 litres of liquid containing the condensed tannins (CT). Approximately $1.5 \mathrm{~kg}$ of pine bark was extracted to yield approximately 10 litres of solution for freeze drying.

The solution obtained was frozen in plastic bags placed in shallow trays $(55 \times 20 \mathrm{~cm})$ to create $1 \mathrm{~cm}$ thick layers for freeze drying. Approximately $200 \mathrm{~g}$ of powder was produced, which contained $35 \%$ condensed tannin, of which $31 \%$ was free, $3.5 \%$ was protein-bound and $0.5 \%$ was fibre-bound. The method used to measure the concentration of $\mathrm{CT}$ in the $\mathrm{PBE}$ was the acetonebutanol method, as described by Terrill et al. (1992). The freezedried extract was re-dissolved in distilled water to give the required concentrations. The solution was stirred on a hotplate at $60{ }^{\circ} \mathrm{C}$ for one hour, and centrifuged at $400 \times g$ for 10 minutes to remove small quantities of insoluble material prior to use of PBE for assays.

Experimental design and sporulation inhibition assay. The faecal samples collected from chickens infected experimentally with pure oocysts of each species of Eimeria were obtained from Avivet Ltd, Palmerston North, New Zealand. The samples were brought to the laboratory in a 50-ml centrifuge tubes containing $2 \%(\mathrm{w} / \mathrm{v})$ potassium dichromate to prevent bacterial degradation of oocysts during transportation. The samples were processed as described by Taylor et al. (1995) with some modifications. Briefly, a 2-g faecal sample was transferred from the centrifuge tube into a glass beaker and mixed with $1 \mathrm{ml}$ of $2 \%$ $(\mathrm{w} / \mathrm{v})$ potassium dichromate, before adding $41 \mathrm{ml}$ of water. The mixture was vortexed for $1 \mathrm{~min}$ and the resulting suspension was strained through double-layered cheesecloth into a beaker. The filtrate was thoroughly mixed before transferring $5 \mathrm{ml}$ to a centrifuge tube and then centrifuged for $5 \mathrm{~min}$ at $1000 \times \mathrm{g}$. After removing the supernatant, the pellet was re-suspended in $5 \mathrm{ml}$ of saturated salt solution. After vortexing for $1 \mathrm{~min}$, a subsample was transferred into a McMaster chamber, using a disposable plastic pipette. The tube was vortexed again before the second chamber of the McMaster slide was filled with a second sub-sample. The preparation was left for 5 min before counting, allowing the oocysts to float up. All the oocysts under the grid of each chamber in the McMaster slide were counted using $\times 10$ objectives and the mean of the two counts was calculated. This process was repeated twice and a count of one oocyst was equivalent to 300 ( $45 \mathrm{ml}$ - the total volume of the original suspension of faeces $/ 0.15 \mathrm{ml}$ - the volume of the sample examined) oocysts/sample (Taylor et al. 1995, Haug et al. 2006). The number of unsporulated oocysts in the original aqueous suspension of faeces was adjusted to one million oocysts per ml.

Oocysts of each strain of Eimeria were exposed to four concentrations of PBE (w/v; 0, 250, 500, and $1000 \mu \mathrm{g} / \mathrm{ml}$ ). Three replications were made for each concentration and the whole experiment was repeated to confirm the results. An in vitro assay was used to evaluate the effect of PBE on the sporulation of coccidian oocysts. In this assay, the unsporulated oocysts were incubated with PBE for $48 \mathrm{~h}$ at $25-29^{\circ} \mathrm{C}$ depending on the species of Eimeria, using water baths to maintain constant temperature. The oocysts were gently aerated with an air pump away from sun light. At the end of the incubation, the sporulated oocysts were washed twice in tap water and stored at $4{ }^{\circ} \mathrm{C}$ until being counted. The number of sporulated and non-sporulated oocysts was counted and the percent sporulation was estimated by counting the number of sporulated oocysts in a total of 100 oocysts. In addition, the numbers of sporocysts within each sporulated oocyst of E. maxima were counted and the numbers of abnormal (in terms of shape and size) sporocysts were counted. The oocysts with 4 sporocysts were considered sporulated regardless the shape and size of the sporocysts. The oocysts were slightly flattened under the pressure of a cover slip to better illustrate morphology. Oocysts and sporocysts were viewed using phase contrast microscopy.

Statistical analysis. Data are presented as the mean \pm S.E.M. A t-test or one-way ANOVA, followed by Tukey's test, was used to detect significance among groups. $\mathrm{P}<0.05$ was considered to be statistically significant.

\section{RESULTS}

It can be seen from Fig. 1A that about $80 \%$ of the oocysts of E. tenella managed to sporulate in the control incubations containing oocysts and tap water whereas in incubations containing 250, 500 and $1000 \mu \mathrm{g} / \mathrm{ml}$ of PBE only $42.6 \%, 24.5 \%$ and $10.9 \%$ of the oocysts were able to sporulate (significant decrease when compared to control incubation; $\mathrm{P}<0.0001$ for all concentrations), respectively. This corresponds to $46.8 \%, 69.4 \%$ and $86.4 \%$ inhibition in sporulation relative to control incubations without PBE (Fig. 1B), respectively. In addition, some PBEtreated oocysts were containing abnormal sporocysts as evidenced by their abnormal sizes and shapes (data not shown) in comparison to those obtained from control incubations. 

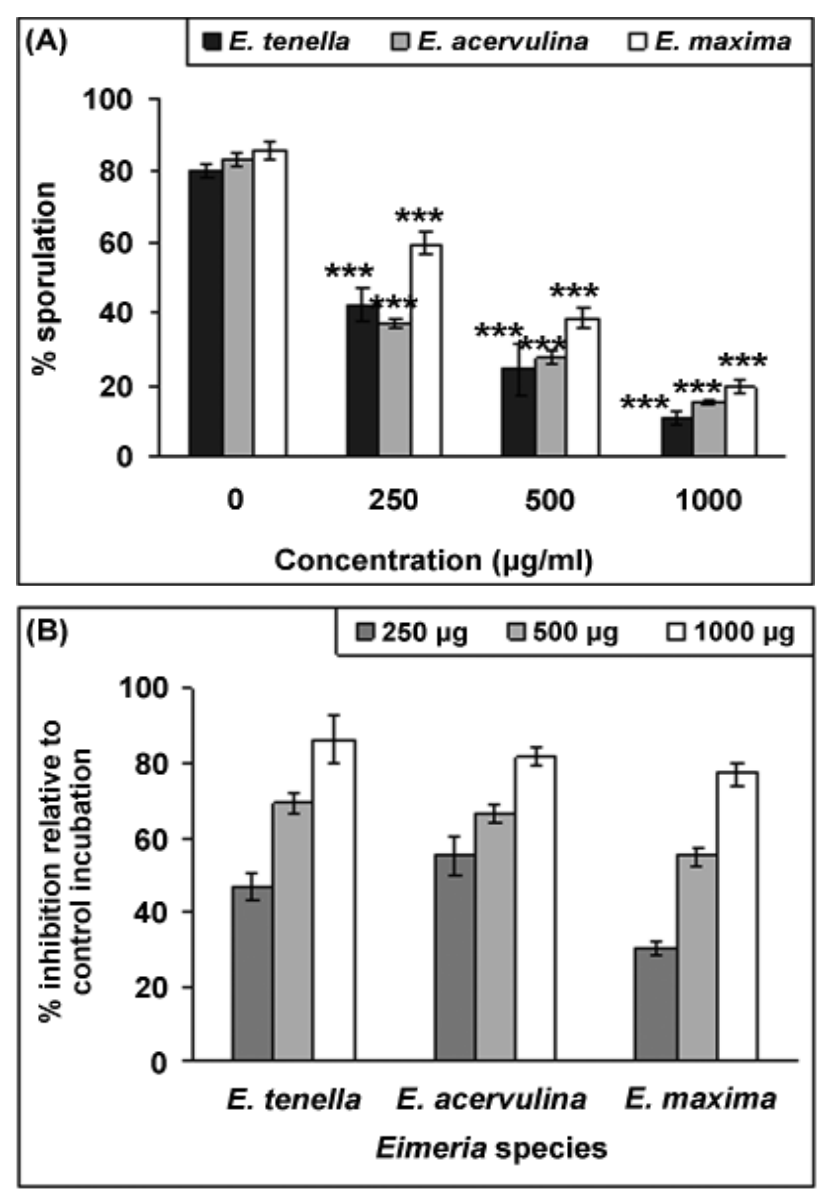

Fig. 1. Percentage sporulation (A) and \% inhibition of sporulation (B) of Eimeria tenella, E. acervulina and E. maxima oocysts recovered from incubations containing water only (control) or different concentrations of pine bark extract. Results are the mean \pm S.E.M. of two independent experiments of triplicate incubations. ${ }^{* * *} \mathrm{P}<0.0001$, level of significance of the inhibitory effect of treated oocysts as compared with that of the untreated oocysts.

In control incubations containing E. acervulina unsporulated oocysts, $83 \%$ of these oocysts were able to sporulate (Fig. 1A). In incubations containing 250, 500 and $1000 \mu \mathrm{g} \mathrm{PBE} / \mathrm{ml}, 37.1 \%, 27.7 \%$ and $15.4 \%$ (significant decrease when compared to control incubation; $\mathrm{P}<$ 0.0001 ) of the oocysts were able to sporulate, respectively. Results shown in Fig. 1B revealed that pine bark treatment at concentrations of 250, 500 and $1000 \mu \mathrm{g} / \mathrm{ml}$ resulted in $55.3 \%, 66.6 \%$ and $81.4 \%$ reduction in sporulation relative to the control incubation.

In another experiment the efficacy of the water extract from pine bark against the oocysts of E. maxima was investigated and the results are presented in Figs. 1 and 2. In control incubation, $85.5 \%$ of the oocysts reached full sporulation whereas in incubations containing 250, 500 and $1000 \mu \mathrm{g} / \mathrm{ml}$ of PBE, 59.5\%, 38.5\% and 19.5\% $(\mathrm{P}<$ 0.0001 relative to control incubation) of the oocysts were able to sporulate, respectively (Fig. 1A). Accordingly, the
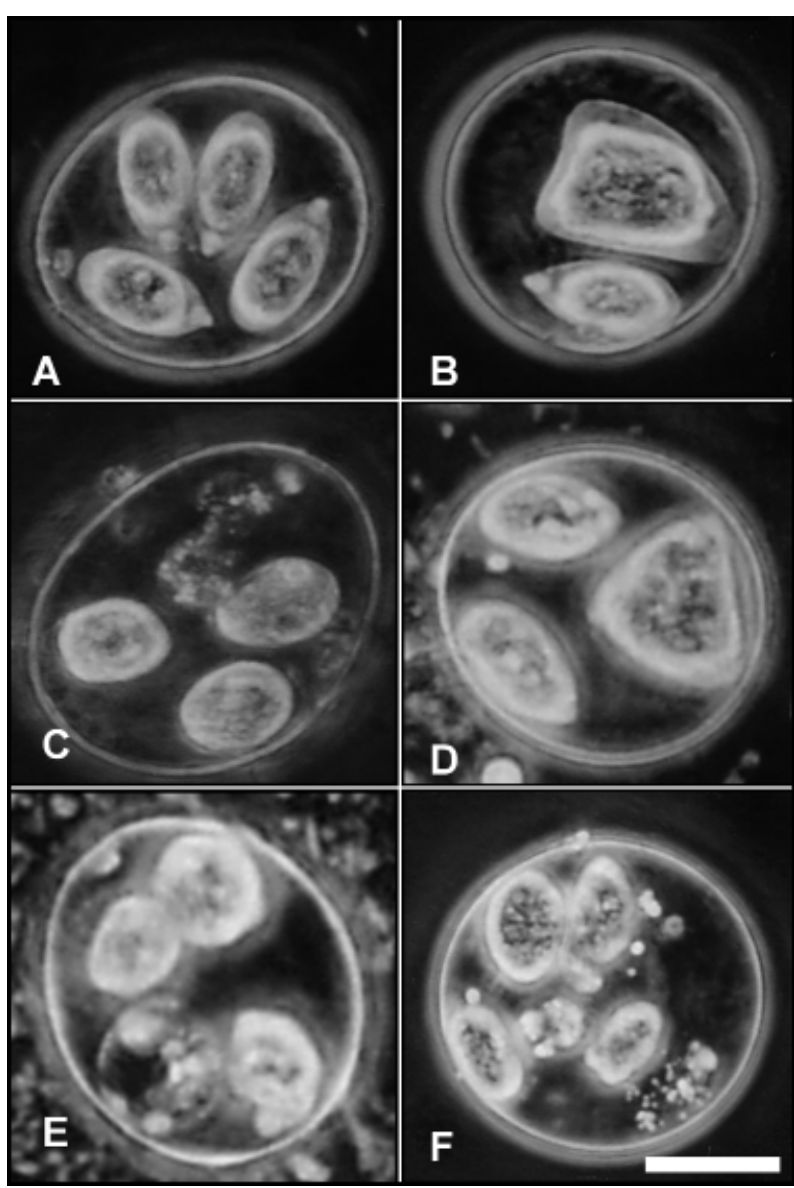

Fig. 2. Photomicrographs of oocysts of Eimeria maxima. A - a typical sporulated oocyst from control incubation containing water only; B-F - abnormal oocysts from incubations containing 500 and $1000 \mu \mathrm{g}$ pine bark extract (PBE)/ml. Oocysts were incubated for $48 \mathrm{~h}$ in the presence or absence of PBE and were viewed under bright field microscopy. Exposure to PBE caused changes in sporocyst numbers, size and morphology. (Note: Oocysts are slightly flattened to better illustrate morphology.) Scale bar $(\mathrm{A}-\mathrm{F})=20 \mu \mathrm{m}$.

exposure of the oocysts of E. maxima to 250,500 and $1000 \mu \mathrm{g} / \mathrm{ml}$ of PBE resulted in $30.4 \%, 54.9 \%$ and $77.2 \%$ reduction in sporulation when compared to the oocysts in the negative control incubation, respectively (Fig. 1B). It is important to mention that E. maxima oocysts were significantly more resistant to the inhibitory action of PBE than the oocysts of E. tenella and E. acervulina.

The photomicrographs of unsporulated oocysts of E. maxima treated for $48 \mathrm{~h}$ with 500 or $1000 \mu \mathrm{g} \mathrm{PBE} / \mathrm{ml}$ and with water are shown in Fig. 2. A normal, but slightly flattened, sporulated oocyst of E. maxima containing four normal sporocysts is shown in Fig. 2A. Up to $12 \%$ of the oocysts exposed to 500 or $1000 \mu \mathrm{g}$ PBE/ml exhibited aberrant sporocysts (Fig. 2B-F). Some oocysts were seen with two (Fig. 2B), three (Fig. 2C, D), four abnormal sporocysts (Fig. 2E) or even more than four sporocysts (Fig. 2F). Although the PBE-treated oocysts retained their outer shapes, their sporonts were contorted into ab- 
normal shapes (Fig. 2B-F). No abnormal sporocysts were observed inside the oocysts recovered from incubations containing $250 \mu \mathrm{g} \mathrm{PBE} / \mathrm{ml}$ or those recovered from the control incubations.

\section{DISCUSSION}

The results show for the first time that water-soluble extracts from pine bark (PBE) containing 35\% condensed tannins have anticoccidial activity as evidenced by their ability to decrease significantly the sporulation of the oocysts of three species of Eimeria, namely E. tenella, E. maxima and E. acervulina, under laboratory conditions. These results are of a major importance as contamination of poultry houses with coccidian oocysts is very difficult to control (Ryley 1980) and all attempts to eradicate infections with Eimeria spp. have failed and hence the negative effects of this parasitic infection persist (Graat et al. 1994). An important factor in the epidemiology of coccidia is the survival of oocysts, which constitute the reservoir of infection. Infection occurs only when chickens ingest sporulated oocysts and infected birds excrete oocysts with their faeces, of which a number will sporulate. Subsequently, a number of sporulated oocysts are ingested by the same or other chickens, hereby spreading the infection. Some studies have indicated that E. tenella oocysts can survive in soil for up to 9 months (Marion and Wehr 1949).

Hur et al. (2005) studied the effects of CT-containing plants on the coccidian infection in goats and found that feeding fresh pine needles and dry oak leaves in combination with lucerne chaff had rapid anticoccidial activities in goats as demonstrated by a sharp decrease in oocyst production. Recently, Jang et al. (2007) studied the anticoccidial effects of green tea-based diets in chickens following oral infection with E. maxima and found that the green tea-fed chicken produced significantly reduced faecal oocysts when compared to the E. maxima-infected group fed standard diet. More recently, Molan and Thomas (2007) studied the effects of aqueous extracts from green tea on the sporulation of E. tenella, E. acervulina and E. maxima and found that addition of $10 \%$ and $25 \%(\mathrm{v} / \mathrm{v})$ of tea extracts to the incubations containing unsporulated oocysts resulted in a significant reduction in sporulation rate. In addition, up to $30 \%$ of the oocysts recovered from incubations containing $25 \%$ of tea extract were with abnormal sporocysts.

The present study showed that E. maxima oocysts were significantly more resistant to the inhibitory action of PBE than the oocysts of E. tenella and E. acervulina. Similarly, Molan and Thomas (2007) reported that the oocysts of E. maxima were more resistant to the action of green tea extract than the oocysts of E. tenella and E. acervulina.

Since CT have been shown to inhibit endogenous enzyme activities (Oh and Hoff 1986, Horigome et al. 1988), then it is possible that the CT from pine bark reduced the proportion of sporulation by inhibiting or inactivating the enzymes responsible for the sporulation process as in helminth eggs (Molan et al. 2003). Jones et al. (1994) suggested that CT may penetrate the cell wall of bacteria and cause a loss of intracellular components. In the present study, the CT from pine bark may penetrate the wall of the oocyst and damage the cytoplasm (sporont) as evidenced by the appearance of abnormal sporocysts in oocysts exposed to 500 and $1000 \mu \mathrm{g} \mathrm{PBE} / \mathrm{ml}$ but not in those recovered from the control incubations or from incubations containing $250 \mu \mathrm{g} \mathrm{PBE} / \mathrm{ml}$. It is now necessary to test the viability and infectivity of PBE-treated coccidian oocysts and this will be the objective of a further study by our group.

\section{REFERENCES}

Ahn J., Grun I.U., Mustapha A. 2007: Effects of plant extracts on microbial growth, color change, and lipid oxidation in cooked beef. Food Microbiol. 24: 7-14

Allen P.C., Fetterer R.H. 2002: Recent advances in biology and immunobiology of Eimeria species and in diagnosis and control of infection with these coccidian parasites of poultry. Clin. Microbiol. Rev. 15: 58-65.

Graat E.A.M., Henken A.M., Ploeger H.W., Noordhuizen J.T.M., VERTOMMEN M.H. 1994: Rate and course of sporulation of oocysts of Eimeria acervulina under different environmental conditions. Parasitology 108: 497-502.

Haug A., Williams R.B., Larsen S. 2006: Counting coccidial oocysts in chicken faeces: a comparative study of a standard McMaster technique and a new rapid method. Vet. Parasitol. 136: 233-242.

Horigome T., Kumar R., OKamoto K. 1988: Effects of condensed tannins prepared from leaves of fodder plants on digestive enzymes in vitro and in the intestine of rats. Brit. J. Nutr. 60 : 275-285.
Hur S.N., Molan A.L., CHa J.O. 2005: Effects of feeding condensed tannin-containing plants on natural coccidian infection in goats. Asian-Austral J. Anim. Sci. 18: 1262-1266.

JAng S.I., Jun M.H., LillehoJ H.S., Dalloul R.A., Kong I.K., Kim S., Min W. 2007: Anticoccidial effect of green tea-based diets against Eimeria maxima. Vet. Parasitol. 144: 172-175.

Jones G.A., McAllister T.A., Muir A.D., Cheng K.J. 1994: Effects of sainfoin (Onobrychis vicifolia Scop.) condensed tannins on growth and proteolysis by four strains of ruminal bacteria. Appl. Environ. Microbiol. 60: 1374-1378.

Kim Y.M., Jeong Y.K., Wang M.H., Lee W.Y., Rhee H.I. 2005: Inhibitory effect of pine extract on $\alpha$-glucosidase activity and postprandial hyperglycemia. Nutrition 21: 756-761.

Kim Y.M., Wang M.H., LeE W.Y., Rhee H.I. 2004: A novel $\alpha$-glucosidase inhibitor from pine bark. Carbohydr. Res. 339: 715-717.

Lillehou E.P., Yun C.H., Lillehou H.S. 2000: Vaccines against the avian enteropathogens, Eimeria, Cryptosporidium and Salmonella. Anim. Health Res. Rev. 1: 47-65. 
Marion M.F., Wehr E.E. 1949: Survival of Eimeria acervulina, E. tenella and E. maxima oocysts in soil under various field conditions. Ann. Trop. Med. Parasitol. 52: 468-472.

Millar C.I. 1999: Evolution and biogeography of Pinus radiata, with a proposed revision of its quarternary history. N. Z. J. For. Sci. 29: 335-365.

Molan A.L., Meagher L.P., Spencer P.A., Sivakumaran S. 2003: Effect of flavan-3-ols on in vitro egg hatching, larval development and viability of infective larvae of Trichostrongylus colubriformis. Int. J. Parasitol. 33: 1691-1698.

Molan A.L., Thомаs D.V. 2007: Green tea water extracts inhibit the sporulation of Eimeria oocysts in vitro. Advancing Poultry Production - Proceedings of the Massey Technical Update Conference 9: 89-97.

Oн H.I., Hoff E. 1986: Effect of condensed grape tannins on the in vitro activity of digestive proteases and activation of their zymogens. J. Food Sci. 51: 577-580.

Packer L., Rimbach G., Virgili F. 1999: Antioxidant activity and biologic properties of a procyanidin-rich extract from pine (Pinus maritima) bark, Pycnogenol. Free Radic. Biol. Med. 27: 704-724.

Porter L.J. 1974: Extractives of Pinus radiata bark. N. Z. J. Sci. 17: $213-218$

Rice-Evans C.A., Miller N.J., Pagana G. 1996: Structure-antioxidant activity relationship of flavonoids and phenolic acid. Free Radic. Biol. Med. 20: 7933-7956.

Received 4 June 2008
RYLey J.F. 1980: Recent development in coccidian biology; where do we go from here? Parasitology 80: 189-209.

Scotт C.W. 1960: Pinus radiata. FAO Forestry and Forest Products Studies, No. 14. FAO, Rome, Italy, 328 pp.

Taylor M., Catchpole J., Marshal R., Norton C.C., Green J. 1995: Eimeria species of sheep. In: J. Eckert, R. Braun, M.W. Shirley and P. Coudert (Eds), COST 89/820, Biotechnology, Guidelines on Techniques in Coccidiosis Research, European Commission, Luxembourg, pp. 25-39.

Terrill T.H., Rowan A.M., Douglas G.B., Barry T.N. 1992: Determination of extractable and bound condensed tannin concentrations in forage plants, protein concentrate meals and cereal grains. J. Sci. Food Agric. 58: 321-329.

WALDENSTEDT L. 2003: Effect of vaccination against coccidiosis in combination with an antibacterial oregano (Origanum vulgare) compound in organic broiler production. Acta Agric. Scand. Anim. Sci. 53: 101-109.

Williams R.B. 2006: Tracing the emergence of drug-resistance in coccidia (Eimeria spp.) of commercial broiler flocks medicated with decoquinate for the first time in the United Kingdom. Vet. Parasitol. 135: 1-14.

Yun C.H., Lillehoj H.S., Lillehoj E.P. 2000: Intestinal immune responses to coccidiosis. Dev. Comp. Immunol. 24: 303-324.

Accepted 9 September 2008 